\title{
FRAGMENTATION OF THE PRIMORDIAL GAS CLOUDS
}

\author{
H. UEHARA ${ }^{1}$, H. SUSA ${ }^{2}$, R. NISHI ${ }^{3}$, M. YAMADA ${ }^{4}$, \\ AND T. NAKAMURA ${ }^{5}$ \\ Dep. of Physics, Kyoto Univ. ${ }^{1,2,3,4}$, \\ Yukawa Institute for Theoretical Physics ${ }^{5}$ \\ Kitashirakawa, Kyoto City 606-01 Japan
}

We discuss the fragmentation of primordial gas clouds in the universe after decoupling. Comparing the timescale of collapse $\left(t_{d y n}\right)$ with that of fragmentation $\left(t_{\text {frag }}\right)$, we obtained the minimum mass of a fragment analytically as following way.

We consider the fragmentaiton of the cylidrical cloud which consists of hydrogen molecules. The condition for the collapsing cloud to fragment is $t_{d y n} \sim t_{\text {frag }}$ and, for the cylindrical cloud which collapses isothermally, it becomes $t_{d y n} \sim(\gamma-1) t_{\text {cool }}$, where $t_{\text {cool }}$ is the cooling timescale. Each timescales are estimated as follows,

$$
t_{\text {frag }} \sim \frac{1}{\sqrt{G \rho}}, \quad t_{c o o l} \sim \frac{\frac{1}{\gamma-1} \frac{M}{\mu m_{\mathrm{H}}} k_{B} T}{2 \pi R \sigma T^{4} \frac{\Delta \nu}{\nu} \alpha_{c}},
$$

where $\Delta \nu / \nu=v_{\mathrm{H}_{2}} / c=k_{B} T / m_{\mathrm{H}} c^{2}, k_{B} T=\mu m_{\mathrm{H}} G M / 2$, and $M, R, T$ are the line density, radius, temperature of the cylindrical cloud and $\alpha_{c}$ is the effective number of line emissions. From the above equations we obtain

$$
M_{\text {frag }} \sim 2 \pi R M \sim \sqrt{\frac{1}{\alpha_{c}}} \frac{1}{\mu^{9 / 4}} \frac{m_{P l}^{3}}{m_{\mathrm{H}}^{2}},
$$

where $m_{P l}=\sqrt{h c / G}$ is the Planck mass.

Above estimate shows that the minimum mass of a fragment of the primordial gas cloud is essentially determined by the Chandrasekhar mass.

\section{References}

Uehara, H., Susa, H., Nishi, R., Yamada, M. and Nakamura, T. (1996) Fragmentation of the Primordial Gas Clouds and the Lower Limit on the Mass of the First Stars, Astrophysical Journal, Vol. 473, pp.L95-L98 